\title{
Prölss/Martin, Versicherungsvertragsgesetz
}

\author{
Inhaltsübersicht
}

\author{
A. Geschichte und Personalia \\ I. Der Autor Erich Prölss \\ II. Fortführung durch Anton Martin und Jürgen Prölss \\ III. Die Zeit nach Anton Martin \\ B. Der Kommentar selbst \\ I. Konzeption und Stil \\ II. Inhalt \\ C. Die Zusammenarbeit der Autoren
}

\section{A. Geschichte und Personalia}

\section{Der Autor Erich Prölss}

Der von Erich R. Prölss (1907-1969) begründete Kommentar zum VVG hat eine ziemlich lange Geschichte. Sie beginnt 1935, als der Beck-Verlag die 1. Auflage herausbrachte, obwohl 1932 dort schon ein VVG-Kommentar von Otto Warneyer erschienen war. Man traute dem neuen Kommentar offenbar mehr zu als dem Warneyerschen, der dann auch nicht mehr neu aufgelegt wurde. Dieses Vertrauen erwies sich als gerechtfertigt: Bis zum Ende des Zweiten Weltkrieges erschienen noch drei Auflagen. Trotz Schwierigkeiten bei der Papierbeschaffung war der Kommentar Ende 1948 schon wieder in 5. Auflage auf dem Markt. Danach erschienen aus der Hand des Begründers des Kommentars noch zwölf weitere Auflagen, die letzte 1968, kurz vor seinem Tod.

Erich R. Prölss, der Vater des Verfassers dieses Beitrags, war zu der Zeit, als er die 1. Auflage schrieb, Rechtsanwalt. Sein Wunsch, in den Staatsdienst einzutreten, wurde wegen der damaligen Wirtschaftslage und den daraus resultierenden Einstellungsbeschränkungen nicht erfüllt, obwohl er im Alter von 24 Jahren das Assessorexamen mit ,sehr gut“ bestanden hatte.

1938 wurde er aber Mitglied des Vorstandes der - heute nicht mehr als selbständiges Unternehmen tätigen - Bayerischen Rückversicherungs AG und bald darauf dessen Vorsitzender. Das Kommentieren betrieb er also immer neben einem äußerst anstrengenden und anspruchsvollen Hauptberuf, der nach seiner Berufung in den Vorstand der „Bayerischen Rück“ auch nicht mehr allzuviel mit Jurisprudenz zu tun hatte. Es war freilich eine seiner Leidenschaften, der er abends und an Wochenenden frönte. Fremde Hilfe nahm er kaum in Anspruch, wenn man von rein technischen Dingen absieht. 
Die Arbeitsweise von Erich Prölss war - gemessen an heutigen Verhältnissen geradezu primitiv. Sein „Computer“ bestand aus einem Zettelkasten, der sich zu einem Ungetüm von Kartothek auswuchs, weil alte Karteikarten nicht einfach weggeworfen, sondern nur ihres grünen „Reiters“ beraubt wurden, der sie als aktuell kennzeichnete. Die Karten enthielten Auszüge aus Urteilen, Aufsätzen und Büchern. Sie wurden von einer Sekretärin nach stenographischen Vorlagen getippt und häufig danach noch mit stenographischem Gekritzel versehen; denn Erich Prölss war ein begnadeter Stenograph und auf äußerste Arbeitsökonomie bedacht. Das Anschwellen des ,Zettelkastens“ beruhte auch darauf, daß er - insbesondere im Hinblick auf Gerichtsentscheidungen - geradezu materialsüchtig war. Selbst jedes Amtsgerichtsurteil, dessen er habhaft werden konnte, ,,verzettelte“ er. Die gesamte österreichische Rechsprechung zum österreichischen VVG, das damals im wesentlichen dem deutschen entsprach, wurde erfaßt. Nach der Teilung Deutschlands durchforstete er sogar die Publikationsorgane der früheren DDR auf Entscheidungen hin, die auch für das bundesrepublikanische Recht interessant sein konnten, und zitierte solche, was nicht überall auf Zustimmung stieß, zumal da er DDR nicht in Anfuihrungsstriche setzte. Diese aufwendige Materialschlacht bedeutete natürlich nicht, daß alle „Eroberungen“ Eingang in den Kommentar fanden. Vielmehr war er auf äußerste Kürze bedacht. Deshalb überlegte er sehr genau, was unter den Tisch fallen konnte.

Rechtswissenschaftlich arbeitete Erich Prölss nicht rechtsgebiets-, sondern gegenstandsbezogen. Das heißt: Er wandte sich nicht einem bestimmten Rechtsgebiet zu, sondern einem bestimmten Phänomen - der Versicherung -, das er aus der Perspektive verschiedenster Rechtsgebiete durchleuchtete. Daher schrieb er auch einen Kommentar zum VAG (1. Auflage 1955) und befaßte sich in seinen Veröffentlichungen etwa auch mit steuer- und bilanzrechtlichen Fragen.

Sein die Versicherung in allen ihren Facetten erfassender Blick bedeutete auch, daß er sich nicht innerhalb des Versicherungsvertragsrechts spezialisierte, sondern die Vorschriften zu sämtlichen Versicherungszweigen selbst und ohne fremde Hilfe kommentierte. Hinzu kamen eher unangenehme Materien wie etwa nach der Währungsreform (1949) der Einfluß der Währungsumstellung auf Versicherungsverträge oder - nach der Teilung Deutschlands - das ,,interzonale“ Versicherungsvertragsrecht.

Die ersten Auflagen des Kommentars erschienen in der Zeit des Dritten Reiches. Da Erich Prölss alles andere als ein Nationalsozialist war (und - obwohl ,Wirtschaftsführer“ - nicht einmal ,formell“ Mitglied der NSDAP wurde), blieb auch der Kommentar von nationalsozialistischem Gedankengut verschont. Das schließt nicht aus, daß Urteile zitiert wurden, die ihrerseits nationalsozialistisch gefärbt waren.

Das Verhältnis von Erich Prölss zum Beck-Verlag, repräsentiert durch den damaligen Inhaber des Verlages, Dr. Dr. h.c. Heinrich Beck und durch den Cheflektor Dr. Höller, einem ausgesprochen schöngeistigen Mann, war sehr gut. Allerdings war Erich Prölss - das sei nicht verschwiegen - in mancher Hinsicht auch ein Quälgeist für den Verlag. So war er versessen darauf, daß der Kommentar ständig die „,neuesten Nachrichten“ wiedergebe. Am liebsten wäre ihm sozusagen eine monatliche Ergänzungslieferung gewesen. Daher versuchte er, jede Auflage mög- 
lichst niedrig zu halten, und handelte mit dem Verlag aus, daß er den Druck einer Neuauflage verlangen konnte, wenn eine bestimmte Stückzahl der alten Auflage verkauft war. Ein weiterer Punkt war das Autorenhonorar. Erich Prölss hatte erfahren, daß einige andere Autoren einen höheren Prozentsatz des Verkaufspreises erhielten als er. Das ließ ihm keine Ruhe. Dabei ging es ihm gar nicht um das Geld. Aber es kränkte sein Selbstbewußtsein, daß er trotz seiner Leistungen schlechter behandelt wurde als andere. Diese Empfindlichkeit hatte ihre Wurzel in seiner Jugend.

\section{Fortführung durch Anton Martin und Jürgen Prölss}

Nach dem Tod von Erich Prölss (1969) betraute der Verlag Dr. Anton Martin und den Verfasser mit der Fortführung des Kommentars. Martin war als Mitglied der Rechtsabteilung der „Allianz“ (später deren stellvertretender Leiter) ${ }^{1}$ ein willkommener Praktiker, der durch diverse Veröffentlichungen auch seine wissenschaftliche Befähigung unter Beweis gestellt hatte. Der Verfasser war promovierter Assistent von Karl Larenz. Er war ebenfalls schon schriftstellerisch hervorgetreten und von seinem Vater in einige Geheimnisse des Versicherungsvertragsrechts eingeweiht worden. Martin interessierte sich vor allem für die Sachversicherung und die damit besonders verknüpften allgemeinen Vorschriften, der Verfasser für die „ganz“ allgemeinen Bestimmungen (ausgenommen die $\S \$ 43-48$ ) und die Vermögensschadenversicherung, insbesondere die Haftpflichtversicherung. Dies war der Ausgangspunkt für die Verteilung des Stoffes. Martin übernahm zunächst auch die gesamte Personenversicherung. Später ging die Lebensversicherung an den Verfasser.

Martin und der Verfasser waren ein eigenartiges Team. Die Charaktere unterschieden sich - jedenfalls vordergründig - extrem, was auch zutage trat, weil beide kein Blatt vor dem Mund nahmen. Trotzdem (oder deswegen?) wurde der Kontakt, auch der persönliche, ziemlich intensiv und machte - jedenfalls hintergründig - Freude. Allerdings gab es auch Turbulenzen.

Für Martin, der gerne die wissenschaftliche Laufbahn eingeschlagen hätte, war der Verfasser einerseits ein „Vatersöhnchen“, andererseits als Larenz-Assistent aber auch respektabel und zugleich beneidenswert. Das war nicht schwer zu durchschauen, und so schlug der Verfasser gleich einen offenen Ton an, um irgendwelche Ressentiments auszuschalten. Martin - Freund einer schon ans Brutale grenzenden Offenheit - gefiel dies. Er akzeptierte den Verfasser und wurde mit der Zeit geradezu zutraulich, was angesichts seiner ,knorrigen“ Art erstaunte.

Martin, der mit einem zivilprozeßrechtlichen Thema promoviert wurde, war ein extrem scharfsinniger und origineller Jurist. Er stieß ständig auf neue oder jedenfalls in Vergessenheit geratene Probleme und interessierte sich auch intensiv für Fragen, die dem Verfasser Kopfzerbrechen bereiteten. Da auch der Verfasser Diskussionen liebte, verging fast kein Monat ohne lange Telephonate. Um ein Pro-

\footnotetext{
${ }^{1}$ Er lehnte es ab, Leiter der Abteilung zu werden, weil dies einen teilweisen Abschied vom Versicherungsvertragsrecht bedeutet hätte.
} 
blem zu illustrieren, wartete Martin oft mit skurrilen Fällen auf, zu deren müheloser Erfindung ihn seine reiche und wohltrainierte Phantasie befähigte. Eines seiner Lieblingsthemen war das versicherbare und versicherte Interesse in all seinen Facetten. Dies brachte es mit sich, daß er sich schon früh zu der Ansicht bekannte, daß fremde Haftpflichtinteressen in weitem Umfang durch die Sachversicherung des Eigentümers geschützt sind. Das blieb nicht ohne Folgen. ${ }^{2}$

Dem Verfasser gefiel der schnörkellose Umgang, den Martin mit Menschen und Dingen pflegte. Die erwähnten Turbulenzen hatten ihren Grund vor allem in den unterschiedlichen Arbeitsweisen. Martin brachte sein Manuskript während seiner Bürozeit - sein Arbeitgeber gestattete ihm dies ausdrücklich - laufend auf den neuesten Stand. Demgegenüber bevorzugte der Verfasser das „Hauruck-Verfahren“", d.h.: er konzentrierte sich erst auf die Bearbeitung der Neuauflage, wenn eine solche in absehbarer Zeit drohte, weil er glaubte, die ständige Beschäftigung mit dem Kommentar würde ihn von seinen Aufgaben als Assistent und Habilitand und später als Hochschullehrer zu sehr ablenken. Das Risiko dieser Arbeitsweise besteht natürlich darin, daß man die bis zum Termin der Abgabe des Manuskripts verbleibende Zeit überschätzt. Der Verwirklichung dieses Risikos entging auch der Verfasser nicht, was verständlicherweise den Unmut Martins hervorrief, der fast zu jeder Zeit ein aktuelles Manuskript präsentieren konnte. Dieser Unmut ging ein Mal so weit, daß Martin dem Verfasser androhte, diesem seine Gunst zu entziehen (und sogar: den Verfasser beim Inhaber des Verlages ,,anzuschwärzen“), wenn er den Termin nicht einhalte. Das hatte zur Folge, daß der Verfasser kurz vor diesem Termin mit Hilfe von Kaffee und Zigaretten fast drei Tage ohne jeden Schlaf durcharbeitete. Das Erstaunen Martins darüber, daß der Verfasser mit dem Manuskript bewaffnet zur vereinbarten Zeit im Verlagsgebäude erschien, entschädigte für die Entbehrungen.

Martin, der 1982 auch einen umfänglichen Kommentar zur Sachversicherung im Beck-Verlag veröffentlicht hatte, ${ }^{3}$ starb 1990. Die letzte Auflage des VVGKommentars, deren Erscheinen er noch erlebte, war die 24. (1988).

\section{Die Zeit nach Anton Martin}

Die dritte Phase des Kommentars begann noch zu Lebzeiten Martins. Das zu berücksichtigende Material wuchs und wuchs. Zwei Verfasser konnten seine Bearbeitung innerhalb tolerabler Fristen nicht mehr schaffen. So wurde Ausschau nach Mitautoren gehalten. Eine schwierige Sache. Diverse Namen fielen und wurden wieder verworfen. Schließlich richtete der neue Lektor des VVG-Kommentars, Christian Schopp, sein bekannt „,markterfahrenes“ Auge auf Ulrich Knappmann und Wolfgang Voit, Richter an einem versicherungsrechtlichen Senat des OLG Hamm, dessen Vorsitz damals Voit (später Knappmann) führte. Beide ausgepichte Praktiker, die mit allen Wassern des Versicherungsvertragsrechts gewaschen waren und auch die Lupe der Theorie nicht verschmähten. Sie stießen auf allgemeine Zustim-

\footnotetext{
2 Siehe unten S. 590.
}

${ }^{3}$ Die 3. Auflage (1992) wurde aus seinem hinterlassenen Manuskript bestritten. 
mung, und die Freude war groß, als sie ihre Bereitschaft erklärten, an dem Kommentar mitzuwirken.

So wurden die Karten neu verteilt. Knappmann bearbeitete die $\S \S 35-39,41$, 42, 158b-k, 179-185, Voit die $\S \S 62-66,129-158$ a. Auch für die Kommentierung der AVB änderten sich die Zuständigkeiten. Das neue Team brachte die 24. Auflage heraus. Aber auch nicht mehr; denn Martin starb vor Erscheinen der 25. Auflage. Wieder begann die Suche nach einem neuen Autor, der möglichst auf der Hochschullehrerbank sitzen sollte. Die Wahl fiel auf Helmut Kollhosser, renommierter und „einschlägig“ hervorgetretener Professor an der Universität Münster, der sie - Gott sei Dank - annahm. Kollhosser bearbeitete die Gebiete, die Martin verblieben waren, und die Lebensversicherung. Unter der Ägide der Autoren Knappmann, Kollhosser, Jürgen Prölss und Voit erschienen die 25. (1992) und die 26. (1998) Auflage. Die 26. Auflage machte besonders viel Mühe, weil die VVG-Reform von 1994 eingearbeitet werden mußte. Die Kommentierung der neuen Vorschriften zur Rechtsschutz- und Krankenversicherung ( $§ 1581-158$ o, $178 \mathrm{a}-\mathrm{n})$ übernahm der Verfasser.

Bevor die 27. Auflage (2004) erschien, starb Voit am 6. Mai 2002. Gleichwohl hat er die 27. Auflage noch mitverfaßt; denn er hinterließ ein Manuskript, das Knappmann auf den neuesten Stand brachte. Kollhosser erlebte das Erscheinen der 27. Auflage zwar noch, starb aber bald danach (30.12. 2004). Das waren (und sind) herbe Verluste, die einen als Mitautor auch dann persönlich treffen, wenn man zu den Verstorbenen nur eine mehr oder weniger sachliche Beziehung hatte. Immerhin gibt es einen Lichtblick: Christian Armbrüster (Freie Universität Berlin), ein Schüler des Verfassers, übernahm schon vor Erscheinen der 27. Auflage die Fortführung des Kommentars zur Rechtsschutzversicherung und zum EGVVG.

\section{B. Der Kommentar selbst}

\section{Konzeption und Stil}

Erich Prölss wollte einen kurzen Kommentar für Praktiker schreiben, der, wie er sagte, auch in einer kleinen Aktentasche Platz findet. ${ }^{4}$ Für so etwas war er auch prädestiniert, da er ein Meister knapper und präziser Formulierungen war. ${ }^{5}$ Hinzu kam, daß ihm der Verlag Vorgaben im Hinblick auf den Umfang einer Neuauflage machte: dieser dürfe so und so viele Seiten nicht überschreiten. Dieser Wille zur gedrängten Kürze und deren ,objektive“ Notwendigkeit bedeutete, daß etwas langwierigere Argumentationen unterblieben und insofern auf die Literatur, soweit vorhanden, verwiesen wurde. Nicht selten hatte ja auch Erich Prölss selbst der Wissenschaft, der er keineswegs abhold war, außerhalb des Kommentars gefrönt.

\footnotetext{
${ }^{4}$ Noch die 17. Auflage, die letzte von ihm bearbeitete, hatte - trotz kleinen Formats - nur 1090 Seiten. Damit betrug ihr Umfang das Zweieinhalbfache der 1. Auflage (ca. 430 Seiten).

${ }_{5}$ Als Freund von Sprachspielereien aller Art verfaßte er auch Schüttelreime und war stolz, den kürzesten aller Schüttelreime präsentieren zu können: „Anna aß Ananas“. Ob Erich Prölss wirklich der erste war, dem dieser Reim einfiel, bleibe offen. Jedenfalls hat auch er ihn erfunden.
} 
Das änderte sich - zunächst kaum sichtbar -, als Martin und der Verfasser den Kommentar übernahmen. Peu à peu wurde die Argumentation angereichert. Zwar verwandelte sich der Holzschnitt damit noch nicht in eine Miniatur, aber ein wenig ging es in diese Richtung und diese wurde beibehalten, als die neuen Mitautoren auf den Plan traten. Auch der Verlag war beim Umfang der Neuauflagen nicht mehr so engherzig. Da sich überdies die in der Praxis auftauchenden Probleme, die zumindest einem auf eine gewisse Vollständigkeit bedachten Kommentatorenblick als berücksichtigenswert erschienen, wie die Kaninchen vermehrten, wuchs sich das Buch zu einem ,,mittleren“ Kommentar aus. Der Umfang der 27. Auflage beträgt fast 2700 Seiten, wobei das Format schon vier Fünftel des „Palandt-Formates“ erreicht. Mit dem Gargantua unter den VVG-Kommentaren, dem „Bruck/Möller“, kann sich das natürlich nicht messen.

Das Anwachsen des „Prölss/Martin“ hatte für den Verlag auch einen Vorteil: Die Stelle eines ,,wirklichen“ Kurzkommentars zum VVG wurde frei und konnte daher mehr oder weniger ,konkurrenzfrei“ besetzt werden. Und so erblickte der „Römer/Langheid“ 1997 das Licht der Welt, der inzwischen schon in 2. Auflage erschienen ist.

\section{Inhalt}

Gegenstand des Kommentars sind nicht nur das VVG und das EGVVG. Auch das österreichische VVG wird nach wie vor berücksichtigt, obwohl es sich inzwischen vom deutschen nicht unerheblich unterscheidet. Das Besondere an dem Werk ist aber, daß von Anfang an auch die wichtigsten AVB kommentiert worden sind. Zunächst fand sich deren Erläuterung in Anhängen zu VVG-Bestimmungen. Das führte zu einer starken Unübersichtlichkeit, so daß der Kommentierung der AVB später ein eigener Teil gewidmet wurde, dessen Umfang den der Kommentierung des VVG jetzt um ca. 500 Seiten übersteigt und ca. 60\% des Gesamtvolumens ausmacht.

Diese umfängliche Berücksichtigung der AVB ist natürlich in erster Linie darauf zurückzuführen, daß die Zahl der - von diversen Publikationsorganen ans Licht gezerrten - Urteile und der wissenschaftlichen Veröffentlichungen zu den AVB wächst und wächst. Früher gab es nur wenige AVB, deren Kommentierung eines fettleibigen Zettelkastens bedurfte, um in der Sprache altmodischer Arbeitsweise $\mathrm{zu}$ bleiben. Star in dieser Hinsicht waren die AKB. Das hat sich gründlich geändert. AVB, die früher ein Dornröschendasein führten, sind aus ihrem Schlummer erwacht. Das gilt z.B. für die Rechtsschutz- und Krankenversicherungsbedingungen, deren Kommentierung früher nur weniger Seiten bedurfte, während sie jetzt insgesamt fast 280 Seiten füllt.

Die Zunahme der gerichtlichen Streitigkeiten, die sich um die AVB ranken, beruht vor allem darauf, daß darüber geführte Prozesse, die prima facie nicht gerade als aussichtsreich erscheinen, nicht von vornherein verloren gegeben werden müssen. Die vom BGH praktizierte Auslegung von AVB aus der Perspektive des verständigen Durchschnittsversicherungsnehmers (an Stelle der früher favorisierten „gesetzesähnlichen“ Auslegung) und der Einwand der Unwirksamkeit von AVB 
(auch wegen deren Intransparenz) sind ziemlich scharfe Waffen in der Hand des Versicherungsnehmers geworden, und man kann oft nicht voraussehen, wen die Gerichte zum Gewinner küren. Also kann man als Versicherungsnehmer ruhig einmal einen Prozeß riskieren, zumal da die Hilfe eines prozeßbereiten Rechtsanwalts leicht zu haben ist. Gleichwohl ist die Zunahme von Streitigkeiten in Branchen, in denen es typischerweise nicht um sehr viel Geld geht, wie in der Rechtsschutzversicherung, erstaunlich. Wenn die allgemeine Wirtschaftslage nicht rosig ist, wird eben um jeden Euro gekämpft.

Allgemeine Aussagen über den Inhalt der Kommentierungen lassen sich kaum machen. Nur dreierlei sei gesagt.

1. Veröffentlichungen, die sich mit Rechtsmaterien befassen, welche die Beziehungen zwischen zwei „Lagern“ regeln, wie dies auch beim Versicherungsvertragsrecht der Fall ist, verfolgen manchmal eine von rechtspolitischen Vorstellungen vorgezeichnete „Linie“, die eine Zuordnung zu dem einen oder dem anderen Lager rechtfertigt. Eine solche Linie wird man im „Prölss/Martin“ nicht finden. Er ist in seiner generellen Haltung weder ,,versichererfreundlich“ noch ,,versicherungsnehmerfreundlich“. Das schließt es nicht aus, daß der Kommentar jetzt dem Schutz des Versicherungsnehmers manchmal mehr Aufmerksamkeit schenkt als dies früher der Fall war.

Das heißt aber nicht, daß der Kommentar früher die Partei der Versicherer ergriff. Zwar waren Erich Prölss (als Rückversicherer, dessen Kunden die Direktversicherer waren, und als Mitglied des Aufsichtrates verschiedener Direktversicherer) und Martin (als Syndikus der ,Allianz“) der Versicherungswirtschaft verbunden. Gleichwohl bemühten sie sich um Objektivität. Erich Prölss sah besonders deutlich das Dilemma des Schutzes der Versicherungsnehmer: was man ihnen gibt, bezahlen sie mit höheren Prämien. Der Schutz der Versicherungsnehmer ist also meist mit künftigen Opfern der Versicherungsnehmer verbunden. Wenn Erich Prölss daher in manchen Fragen, deren Beantwortung Spielräume bot, zugunsten der Versicherer entschied, so war dies die Betonung - manchmal auch Überbetonung - der Kehrseite der Medaille und nicht einfach das Bestreben, die Gewinne der Versicherer zu steigern. So wurde er denn auch nicht müde, den Versicherern den Mangel an publikumswirksamer Offenlegung des Dilemmas vorzuwerfen.

Martin war trotz seiner Position ebenfalls neutral. In mancher Hinsicht schlug sein Herz sogar eher für die Versicherungsnehmer. Wenn er von einer versicherungsnehmerfreundlichen Lösung eines Problems überzeugt war, es ihm aber zu gewagt erschien, diese publikumswirksam zu vertreten, brachte er sie manchmal in nebensächlichen Kontexten unter, was zu - von ihm sehenden Auges in Kauf genommenen - Widersprüchen führte. Oder er bat den Verfasser darum, seiner Ansicht, falls der Verfasser sie teilen sollte, irgendwo Unterschlupf zu gewähren.

2. Die Autoren kleben (und klebten) nicht an ihren Ansichten. Sie trennen sich auf Grund besserer Einsicht auch von lieb gewordenen Meinungen, so daß sich des öfteren der Hinweis ,a. M. noch die ... Aufl.“. findet. Der Benutzer des Kommentars kann sich also nie sicher sein, daß in einer Neuauflage nur der neue Stoff 
eingearbeitet wurde und im übrigen alles beim alten geblieben ist. Ein Beispiel aus jüngster Zeit: Aufgabe der von der Rechtsprechung abweichenden Ansicht, daß der schon bei Vertragsschluß verkehrsunsichere Zustand eines Kfz nur unter dem Aspekt der $\S \S 16 \mathrm{ff}$. VVG und nicht dem der $\S \S 23 \mathrm{ff}$. VVG relevant sei. ${ }^{6}$

3. Das Versicherungsvertragsrecht ist zwar ein außerhalb des BGB geregeltes Rechtsgebiet. Dies sollte aber nicht dazu verleiten, es möglichst weitgehend von seinen bürgerlichrechtlichen Wurzeln abzuschneiden. Es war daher immer ein Anliegen des Kommentars, den Sonderrechtscharakter des Versicherungsvertragsrechts zu relativieren und dieses so weit wie möglich als Teil des bürgerlichen Rechts zu begreifen (Beispiel unten).

Hier ist nicht der Ort, diverse ,interessante“ Aussagen des Kommentars zu konkreten Problemen vorzuführen. Es sei nur darauf hingewiesen, daß der Kommentar manchmal auch gegen den Strom schwimmt. So akzeptiert er nach wie vor nicht die fast allgemeine Ansicht, daß die vertraglichen und gesetzlichen Obliegenheiten des Versicherungsnehmers eine eigenständige versicherungsrechtliche Figur darstellten und keine Verhaltenspflichten zum Inhalt hätten. ${ }^{7}$ Auch der angeblich gewohnheitsrechtlichen - Haftung des Versicherungsnehmers für seine „Repräsentanten“ steht er skeptisch gegenüber und bevorzugt eine „gemäßigte“ Anwendung des $\S 278$ BGB. ${ }^{8}$ Im Bereich des $\S 61$ VVG wird jedwede aus dem objektiven Recht hergeleitete Fremdhaftung abgelehnt. ${ }^{9}$

4. Die Gerichte haben meist ein ,gutes“ Verhältnis zu einem Kommentar, der sich durchgesetzt hat, vor allem zu einem Kommentar zu Spezialmaterien. Das heißt: Sie ziehen ihn heran und zitieren ihn dann auch meist. So können sich auch die Autoren des „Prölss/Martin“ kaum darüber beklagen, daß sie von den Gerichten vernachlässigt würden. Schwierig ist es zu beurteilen, ob und inwieweit der Kommentar Gerichtsentscheidungen beeinflußt hat, mögen auch wohlmeinende Stimmen dem Kommentar einen solchen Einfluß attestieren. Auch, wenn ein Gericht die Ansicht eines Autors zustimmend zitiert, läßt sich meist nicht sagen, welches Gewicht die Stimme des Autors bei der Findung des Urteils hatte. Anders ist es, wenn ein Gericht zugunsten einer von einem Autor geäußerten Ansicht von seiner bisherigen Rechtsprechung abweicht. Ein ziemlich seltener Fall. Ein Beispiel für einen solchen Fall ist wohl der - sub specie des Einflusses des Kommentars allerdings etwas „umwegige“ - Umschwung der Rechtsprechung im Hinblick auf den bisher abgelehnten Schutz von Haftpflichtinteressen in der Sachversicherung (BGHZ 145, 393). Martin war, wie schon gesagt, für einen weitreichenden Schutz in den noch von ihm verfaßten Vorbemerkungen zu $\$ 51$ VVG eingetreten. Dieser Ansicht war zunächst kein großer Erfolg beschieden. Sie hat aber Armbrüster dazu veranlaßt, näher über das Problem nachzudenken. ${ }^{10}$ Er kam ebenfalls zu dem Ergebnis eines weitgehenden Schutzes von Haftpflichtinteressen in

6 27. Aufl., § 23 Rn. 5.

7 § 6 Rn. 30.

$8 \S 6$ VVG Rn. $47 \mathrm{ff}$.

$9 \S 61$ VVG Rn. $3 \mathrm{ff}$.

${ }^{10}$ Der Schutz von Haftpflichtinteressen in der Sachversicherung, 1994. 
der Sachversicherung. Seine Ausführungen, denen auch der Verfasser folgte, sind dann maßgeblich an dem Umschwung der Rechtsprechung beteiligt gewesen. Also wohl eine zumindest mittelbare Beeinflussung der Rechtsprechung durch den Kommentar.

\section{Die Zusammenarbeit der Autoren}

Betreuen nur zwei Autoren einen Kommentar, so finden meist regelmäßige Diskussionen statt. Jedenfalls war dies so, als Martin und der Verfasser die einzigen Autoren des Kommentars waren. Das bedeutet zwar keine Gewähr dafür, daß man sich einigt und daher im Hinblick auf Probleme, die die Arbeitsbereiche beider Autoren berühren, eine einheitliche Ansicht vertreten wird. Aber immerhin: Häufig sind verbleibende Divergenzen nicht. Treten mehr als zwei Autoren auf den Plan, so kommt es nur selten zu Diskussionen. Meist weist man einen Mitautor nur darauf hin, daß man eine andere Ansicht als er vertrete, und das Ergebnis ist, daß man sich wechselseitig als ,a. M. ... zitiert. Wenn man einmal davon absieht, daß Diskussionen unter Fachleuten fast immer anregend sind, ist dies auch gar nicht so schlecht. Ein Kommentar der von mehreren Autoren bearbeitet wird, ist eben kein Maschinenteil, das aus einem Guß sein muß. 
https://doi.org/10.17104/9783406731181-583, am 26.04.2023, 05:25:17

Open Access - (cc) EY - http://www.beck-elibrary.de/agb 